\title{
Intermédialités
}

Histoire et théorie des arts, des lettres et des techniques

Intermediality

History and Theory of the Arts, Literature and Technologies

\section{Visages-légendes : de Boris Karloff à Frankenstein}

\section{Johanne Villeneuve}

Numéro 8, automne 2006

Envisager

Facing

URI : https://id.erudit.org/iderudit/1005546ar

DOI : https://doi.org/10.7202/1005546ar

Aller au sommaire du numéro

Éditeur(s)

Centre de recherche sur l'intermédialité

ISSN

1705-8546 (imprimé)

1920-3136 (numérique)

Découvrir la revue

Citer cet article

Villeneuve, J. (2006). Visages-légendes : de Boris Karloff à Frankenstein.

Intermédialités / Intermediality, (8), 173-186. https://doi.org/10.7202/1005546ar
Résumé de l'article

Cet article a comme point de départ le passage du visage « littéraire » du monstre dans Frankenstein de Mary Shelley (1818) au célèbre visage cinématographique qu'a légué à la postérité le film de James Whale sous les traits de Boris Karloff. Le principe de l'inadéquation qui fonde le premier visage est maintenu dans le second, bien que considérablement transforme en fonction des possibilités qu'offre le medium cinématographique et du contexte socioculturel dans lequel celui-ci produit ses effets. Il s'agit de voir comment le Hollywood des années 1930 reprend le vieux filon de la fantasmagorie robertsonienne afin de produire un " flot " particulier de visages monadiques. Sur le mythe de Frankenstein se greffe la légende de Boris Karloff : trajectoire démultipliée d'un visage " sans essence " et vide de son expérience, un visage-cadre légende, portant les marques d'une survivance et d'une errance en partage au détour de la " grande crise ". 


\title{
Visages-légendes: \\ de Boris Karloff à Frankenstein'
}

\author{
Johanne Villeneuve
}

\section{NAISSANCE D'Un VISAgE}

Dans le roman de Mary Shelley, paru en 1818, l'inventeur décrit ainsi sa créature:

With an anxiety that almost amounted to agony, I collected the instruments of life around me, that I might infuse a spark of being into the lifeless thing that lay at my feet. It was already one in the morning; the rain pattered dismally against the panes, and my candle was nearly burnt out, when, by the glimmer of the half-extinguished light, I saw the dull yellow eye of the creature open; it breathed hard, and a convulsive motion agitated its limbs. How can I describe my emotions at this catastrophe, or how delineate the wretch whom with such infinite pains and care I had endeavoured to form? His limbs were in proportion, and I had selected his features as beautiful. Beautiful!-Great God! His yellow skin scarcely covered the work of muscles and arteries beneath; his hair was of a lustrous black, and flowing; his teeth of a pearly whiteness; but these luxuriances only formed a more horrid contrast with his watery eyes, that seemed almost of the same colour as the dun white sockets in which they were set, his shrivelled complexion, and straight black lips. The different accidents of life are not so changeable as the feelings of human nature. I had worked hard for nearly two years, for the sole purpose of infusing life into an inanimate body. For this I had deprived myself of rest and health. I had desired it with an ardour that far exceeded moderation; but now that I had finished, the beauty of the dream vanished, and breathless horror and disgust filled my heart. Unable to endure the aspect of the being I had created, I rushed out of the room ${ }^{2} .$.

1. Je remercie le Conseil de recherche en sciences humaines du Canada qui a permis la réalisation de cette étude, de même qu'Alain Biage pour son assistance, en particulier pour les recherches effectuées dans les archives du New York Times Film Reviews, ainsi que dans le New York Times entre 1926 et 1977.

2. Mary Shelley, Frankenstein; or the Modern Prometheus, Berkeley, Los Angeles, London, University of California Press, 1984 [1818] (reprise de l'édition Pennyroyal de 1983), p. 51-52. Désormais les références au roman seront indiquées par le sigle «F », suivi de la page, et placées entre parenthèses dans le corps du texte. 
Après quoi le docteur va se coucher, troublé par des rêves effrayants dans lesquels sa fiancée apparaît sous les traits du cadavre de sa mère. Il s'éveille, en sueur :

[...] when, by the dim and yellow light of the moon, as it forced its way through the window-shutters, I beheld the wretch-the miserable monster whom I had created. He held up the curtain of the bed; and his eyes, if eyes they may be called, were fixed on me. His jaws opened, and he muttered some inarticulate sounds, while a grin wrinkled his cheeks. He might have spoken, but I did not hear... (F, p. 53-54)

Selon la description qu'en propose le roman, le monstre créé par le docteur Frankenstein a pour visage celui de l'inadéquation : inadéquation de la peau qui laisse transparaître les systèmes musculaires et sanguins; inadéquation entre des aspects du visage (les cheveux noirs et les dents blanches), qui devaient révéler la beauté, et un regard fade, perdu dans la cavité des orbites; inadéquation entre un objet (des yeux) et sa dénomination ( «if eyes they may be called»); inadéquation d'une bouche qui parle mais que l'on n'entend pas. Sur le plan de la chronologie des événements, il s'agit encore de l'inadéquation entre le projet d'une créature prométhéenne et sa réalisation désastreuse. Mais si l'on tient compte de ce qu'un monstre pareil apprend, dans la suite du roman, à parler, à lire et à écrire, l'inadéquation trouve sa résolution dans une mise en forme narrative de l'existence fondée sur l'apprentissage et l'éducation. Devenant peu à peu le sujet de son destin jusqu'à prendre le relais de la narration romanesque, il s'affranchit de ses inadéquations physiques et s'émancipe de son visage. La visagéité du monstre disparaît alors au profit d'une quête narrative, littéraire et existentielle: celle d'une expérience en propre qui passe par l'épreuve éducative et s'achève sur une résolution hautement poétique et spirituelle, alors qu'il est emporté au milieu des glaces de l'Arctique:

"But soon," he cried, with sad and solemn enthusiasm, "I shall die, and what I now feel be no longer felt. Soon these burning miseries will be extinct. I shall ascend my funeral pile triumphantly, and exult in the agony of the torturing flames. The light of that conflagration will fade away; my ashes will be swept into the sea by the winds. My spirit will sleep in peace; or if it thinks, it will not surely think thus. Farewell." (F, p. 237)

Non seulement la créature a-t-elle acquis, au-delà d'un visage, le langage et la science de l'écriture, mais elle se fait nécrologue en rendant à elle-même ses derniers devoirs. La scène finale constitue en effet un acte d'auto-consumation par lequel l'esprit, arrivé à sa pleine détermination, appréhende sa propre extinction: «or if it thinks, it will not surely think thus. » Ainsi, à travers cette auto-poïesis qui culmine dans la sublimation de l'esprit par lui-même, la créature lèguet-elle quelque chose au monde: une exemplarité négative servant à freiner les ambitions faustiennes de l'humanité, mais dont l'issue ne laisse d'exalter la force 
morale de la civilisation. Emporté par les glaces, la créature écrit encore ; telle est la puissance de la «littératie » et, avec elle, celle de la littérature.

On connaît l'immense postérité du monstre, depuis sa naissance littéraire, puis théâtrale, jusqu'à ses multiples reliquats en images: performances cinématographiques, bandes dessinées, figurines de pacotille, caricatures et masques d'Halloween. Au cinéma, la première adaptation du roman, proposée par les studios Edison, date de 19103. Mais la véritable renaissance du monstre survient, bien sûr, avec la prestation de Boris Karloff dans le film de James Whale en 1931, lequel donne à l'histoire de Frankenstein un visage et en fait circuler le nom. La créature ne cesse de rejouer, depuis lors, le mythe d'une vie après la mort marqué par l'extraordinaire popularité d'un visage qui entraîne sa propre disparité, son inadéquation: pourquoi, en effet, le véhicule d'une telle postérité est-il, justement, un visage? Pourquoi une telle prospérité, alors que semblent s'être effacés autour de ce visage les lieux et l'époque qui l'ont vu naître? De quelle histoire participe ce visage?

Dans l'incubateur où vient se loger le visage élaboré par Mary Shelley, un certain nombre d'ingrédients interfèrent jusqu'à le dénaturer et l'arracher entièrement à sa modélisation littéraire et narrative. Seule demeure, dans la version cinématographique, la conception d'une inadéquation. Depuis Shelley, de multiples éléments travaillent en sourdine le visage de la créature et en préparent la spectaculaire reprise cinématographique. Parmi eux, certainement, l'essor d'un fantastique romantique qui réinvestit le gothique littéraire, lui-même tributaire d'une relecture régressive de l'art gothique depuis le célèbre roman d'Horace Walpole auquel on doit le genre littéraire4. Mais au cœur du gothique littéraire, depuis ses premières élaborations jusqu'au début du $\mathrm{Xx}^{\mathrm{e}}$ siècle, se dessine ce que Joëlle Prungnaud appelle « un visage maquillé par les siècles ». Prungnaud s'intéresse pourtant à l'architecture à l'œuvre chez Huysmans. Elle y décrit un art aux prises avec la dégénérescence et la mort, alors que les « ravages du temps sont interprétés comme les symptômes d'un mal physique, d'une infirmité », de sorte que, insiste-t-elle, «l'architecte n'est plus un bâtisseur mais un médecin5 ». Le médecin est déjà à l'œuvre chez Mary Shelley, comme il le sera plus tard chez Stoker, Wells ou Stevenson (Dr. Van Helsing, Dr. Moreau ou Dr. Jekyll). Car il y a derrière chaque monstre un physionomiste au travail. À rebours, le corps

3. Voir Donald F. Glut, The Frankenstein Legend: A Tribute to Mary Shelley and Boris Karloff, Metuchen, New Jersey, Scarecrow Press, 1973.

4. Horace Walpole, The Castle of Otranto. A Gothic Story, 1764. Notons que le roman de Shelley est lui-même contemporain d'une littérature gothique importante. Polodori publie The Vampire en 1819 et Maturin son célèbre Melmoth en 1820.

5. Joëlle Prungnaud, Gothique et décadence, Paris, Honoré Champion, 1997, p. 261. 
humain impose ses ruines, ses bas-reliefs, ses espaces décomposés, ses propres monuments. Mais c'est le cinéma qui, semble-t-il, expose le mieux, par le dévoilement d'un visage, la concrétion d'une telle rencontre entre l'architectural et la médecine, entre la ruine et la couture chirurgicale.

Le visage du monstre donné à voir par Whale est, en ce sens, radicalement différent de celui proposé par le roman: au défaut de consistance que traduit la transparence de la peau à travers laquelle on verrait de manière obscène les veines et les muscles, se substitue, bien au contraire, un surplus de consistance: le visage inadéquat proposé par le film semble fait de pierre et de sutures surexposées. Il est tout à fait significatif que ce qui fasse horreur dans le roman pointe vers une des qualités réputées du cinéma, à savoir la transparence du médium, tandis que le même sentiment dépende, dans le film, de ce qui définit toute textualité, à savoir la consistance d'un tissu, d'une trame. Mais surtout, c'est l'inscription d'un rapport au temps qui afflige le visage cinématographique, au contraire du visage romanesque dont l'importance demeure, du reste, plutôt limitée. L’inadéquation y est schématique et esthétique, mais elle prend dans le film la valeur temporelle d'un visage naissant, quoique déjà usé. L'architecture du visage, pour reprendre le filon gothique, renvoie à des «temps » aussi obscurs qu'étaient évidents les muscles sous la translucidité évoquée par Shelley. Or toute l'entreprise décrite par le roman ne dépend-elle pas, au premier chef, de la mobilisation d'une écriture littéraire capable, justement, de faire preuve de lucidité et de pénétration? Car depuis ses ténèbres, la créature apprendra pourtant à acquérir une conscience, à pénétrer le monde de son esprit jusqu'à la toute dernière clairvoyance dans laquelle il se verra, paradoxalement, abolir. Dans le film, au contraire, le regard du monstre n'est jamais qu'enfoncé en lui-même, engoncé plutôt dans la masse obtuse d'un visage sans figuralité. Le mouvement de pénétration s'abîme dans la prolifération d'un seul visage; la créature s'expose, aussi irradiante que pétrifiée, rappelant sans cesse qu'elle n'est que l'objet d'une science aveuglée par sa volonté de puissance, et non pas le sujet en devenir d'un roman d'apprentissage. Le monstre de Whale n'apprendra rien. Sa courte pérégrination existentielle s'achèvera sur une pantomime sacrificielle sans commune mesure avec l'assomption grandiloquente du personnage littéraire. Lorsque, dans le film de Whale, la porte s'ouvre pour la première fois sur les plans enchaînés de la créature se découvrant enfin à son créateur, c'est la force de cet aveuglement qui irradie l'image. Celle-ci passe, par fondus superposés, d'un plan d'ensemble de la créature à un gros plan de son visage surexposé comme englué de lumière. À la limite, dirions-nous, se perd dans le film, en comparaison du roman, l'idée prométhéenne ou le complexe faustien de la volonté de puissance pour ne laisser place qu'à l'aveuglement seul. L'acuité scientifique a fini par trouver, dans le trop-plein de lumière et de connaissances, son point d'aveuglement; aussi, la 
créature et son créateur commencent-ils, avec le film de Whale, à emmêler leurs identités davantage que leurs destins que confondait le roman jusqu'au périple de l’Arctique. La pénétration optique, qui accompagnait autrefois l'écriture du savant et son art chirurgical s'est révulsée en une matérialisation toute gothique du rapiéçage, du monument empoussiéré et de la massification lourdement exposée. La monumentalité du savoir se perd dans la doublure monumentale de ses matériaux.

Cette résurgence cinématographique de la créature tient encore d'une autre variante du gothique, celle qui se dessine à travers la fantasmagorie de Robertson, une génération précédant le roman de Shelley, et qui consiste à faire apparaître des spectres par les moyens de spectacles optiques, préférablement en des lieux «hantés », tels les châteaux en ruine et les cimetières. À partir des spectacles thématiques de lanternes magiques, jusqu'au «fantascope» (lanterne magique montée sur rail préfigurant les travellings du cinéma), la fantasmagorie se plaît à faire apparaître, entre autres, des têtes coupées. Elle permet la confluence des expérimentations scientifiques et des engouements spiritualistes, d'abord plutôt élitistes, bientôt populaires. Or voici comment Robertson décrivait son expérience:

Aussitôt que je cessais de parler, la lampe antique suspendue au-dessus de la tête des spectateurs s'éteignait, et les plongeait dans une obscurité profonde, dans des ténèbres affreuses. Au bruit de la pluie, du tonnerre, de la cloche funèbre évoquant les ombres de leurs tombeaux, succédaient les sons déchirants de l'harmonica; le ciel se découvrait, mais sillonné en tous sens par la foudre. Dans un lointain très reculé, un point mystérieux semblait surgir : une figure, d'abord petite, se dessinait, puis s'approchait à pas lents, et à chaque pas semblait grandir' ${ }^{6}$.

L'avancée du spectre depuis son tombeau, ou de la créature depuis le lieu suspect de sa fabrication, constitue le mouvement de prédilection de cette cinématographie gothique des années 1930 aux États-Unis. La séquence de l'apparition de Frankenstein, comme celle où apparaîtra Dracula dans la célèbre version inaugurale de Tod Browning, procède exactement de ce mouvement, bien qu'opérée par un montage de plans, car la couture en est toujours la marque indélébile. Du corps entier de la créature à son visage, il doit nécessairement y avoir césure - modalité du spectacle et de sa rampe, caractérisation de la décollation et du visage en tant que singularité qui envahit l'espace. Ce bref effet de montage produit, en réalité, un effet de découpe qui déconcerte et singularise.

Le Hollywood des années 1930 reprend donc le double filon - déjà usé à la corde - de la fantasmagorie robertsonienne et d'une littérature gothique entrée depuis longtemps dans les limbes de la paralittérature. C'est à ce moment

6. Étienne Gaspard Robert, dit Roberston, cité par Max Milner, La fantasmagorie, Paris, Presses universitaires de France, 1982, p. 11. 
que naît le visage qui nous intéresse : celui de «Frankenstein » - du nom doublement étrange de son créateur. Doublement étrange parce que, d’une part, sa consonance longue et brutale offre d'emblée une caricature de l'étrangeté, et d'autre part, le monstre, qui n'a pas de nom chez Shelley, finira par s'approprier à l'usure celui de son créateur. Qui, en effet, pense à l'homme de science en entendant prononcer le nom de «Frankenstein »? Depuis la version cinématographique de Whale, ce nom suppose un décorum; il plante son propre décor; il trahit du masque et scelle une imposture fiduciaire.

\section{UN FLOT DE VISAGES: L'INADÉQUATION DE KARLOFF}

Le cadrage, soutenait Béla Balázs, élimine le visage individuel, lui donne un sens «suprapersonnel7 ». Paradoxalement, ce qui agirait au principe même d'identité, soit le visage, s'émanciperait, au cinéma, de toute individuation ${ }^{8}$. Ce serait là, en effet, l'invention fabuleuse de l'image cinématographique: le détail individué ne s'exprimerait que sous la forme de la démesure. En témoignerait le caractère hyperbolique et monstrueux d'un œil ou d'une bouche accédant aux dimensions de l'écran, mais aussi tout autre objet menu dont la visibilité serait amplifiée et qui, se délestant de son milieu phénoménologique, s’inventerait de nouvelles déterminations et de nouvelles représentations. Ce serait là, finalement, le caractère monstrueux de l'image cinématographique, intéressée par la mimique dévastatrice du visible et ses surgissements hasardeux.

Pourtant, la «démesure » elle-même semble occuper un rôle longuement préparé, patiemment élaboré. Ainsi se construit la prospérité d'un visage, sa prolifération et sa légende, à une époque où, justement, la projection d’un visage ou celle d'un objet en gros plan produit encore l'effet d'une fantasmagorie où se

7. Béla Balázs, L’esprit du cinéma, Paris, Payot, 1977 [1930], p. 149.

8. Le visage y devient le «document» d'une micropsychologie; chacun de ses détails, lorsque montré en gros plan, devient visage à son tour. À telle enseigne que Gilles Deleuze verra dans le gros plan en général la faculté de transformer l'image en visage: l'image, en tant que plaque sensible, réceptive, unité réfléchissante, est affection selon un principe de visagéification; en tant que tendance motrice, la visagéité de l'image constitue un ensemble de traits d'intensité et d'expressions. Deleuze se défait ainsi des derniers encombres de la physiognomonie qui faisaient encore dire à Balázs qu'à l'intérieur du visage se trouvaient «des parcelles de physionomie», derniers postulats, peut-on penser, d'une conception essentialiste du visage. Le visage deleuzien est délibérément machinique, sans essence, décrété par une caméra tantôt humaine, tantôt inhumaine. Voir Gilles Deleuze, Cinéma 1. L'image-mouvement, Paris, Éditions de Minuit, coll. «Critique», 1983 , p. $125^{-172 .}$ 
superposent la décollation et l'apparition. Depuis 1931, où il incarne la créature de Frankenstein9, jusqu'à sa mort en 1969, Boris Karloff porte le fardeau d'un visage qu'il ne serait pas même nécessaire de décrire ici tant sa légende l'a rendu immédiatement visible ${ }^{10}$. Il suffit de voir les films tournés par Karloff au cours de sa carrière, d'en revoir les affiches et les photographies, pour comprendre à quel point le visage de l'acteur et celui du monstre sont devenus indémêlables ${ }^{11}$. Même sous les traits amplifiés et fardés de Fu Manchu (The Mask of Fu Manchu, Charles Barbin, 1932), Karloff a encore ce «je ne sais quoi » qui appartient à Frankenstein. Dans le film The Raven (Lew Landers, 1935), c'est encore le visagelégende frankensteinien qui transpire sous les traits ravagés du pauvre Bateman joué par Karloff. Et si le visage emblématique de la créature laisse transpirer à son tour celui de Karloff, c'est bien parce que la technique du cinéma de l'époque ne peut effacer, comme il en serait d'un simple modèle ou d'une esquisse, la trace phénoménologique de l'acteur. Mais plus complexe est la persistance du visage du monstre dans le visage de l'acteur et des personnages qu'il incarne par la suite. La plupart des différents acteurs qui prennent le relais de Karloff doivent, eux aussi, soumettre leur visage à une désappropriation abyssale : le succès du film de Whale les oblige à se conformer au visage d'un Frankenstein-Karloff. Une telle

9. Karloff a joué le rôle de la créature à deux reprises après sa prestation de 1931: en 1935, dans Bride of Frankenstein (James Whale) et en 1939, dans Son of Frankenstein (Rowland V. Lee).

10. C'est là l'effet même du cinéma et de sa popularité, que de confondre légende et immédiateté, tant l'idée d'une transparence du médium y est largement reçue. Or chez ce visage, la transparence expose ironiquement la masse opaque mais grossièrement travaillée du faciès.

11. Voir Richard Bojarski et Kenneth Beale, Boris Karloff, Paris, Henri Veyrier, 1975; Patrick Brion, Le cinéma fantastique, Paris, Éditions De la Martinière, 1994; Michael Brunas, John Brunas et Tom Weaver, Universal Horrors - The Studios Classic Films 19311946, Jefferson, North Carolina, Mc Farland \& Company, 1990; Ivan Butler, Horror in the Cinema, 3 édition révisée, South Brunswick et New York, A.S. Barnes and Co., 1979; Laurent Arkin, «Petits arrangements avec les acteurs morts», Vertigo, n ${ }^{\circ}$ 11-12, 1994, p. 115-117; Leslie Halliwell, The Dead that Walk - Dracula, Frankenstein, the Mummy and other Favorite Movie Monsters, New York, Continuum, 1988 [1986]; Michael Sevastakis, Songs of Love and Death - the Classical American Horror Film of the 1930s, Westport, Greenwood Press, 1993; Doug Tomlinson et al., Actors on Acting for the Screen-Roles and Collaboration, New York, London, Garland Publisher, 1994; Donald F. Glut, The Frankenstein Legend, Actes du colloque «Frankenstein - Littérature/cinéma », Liège, Éditions du CÉFAL, Les cahiers des paralittératures, nº 7, 1997. 
tendance imprègne jusqu'aux adaptations plus récentes, au cinéma mais également à la télévision et en bandes dessinées ${ }^{12}$.

Ce «je ne sais quoi » qu'inaugure le personnage créé par Karloff touche au visage seul. La créature s'impose en effet par cette contamination d'un visage dans lequel semblent pouvoir se couler tous les visages d'acteurs. Au moment où Karloff connaît la célébrité, Bela Lugosi incarne à l'écran cet autre revenant de l'imaginaire gothique qu'est le vampire Dracula ${ }^{13}$. Le personnage créé par Lugosi connaît sensiblement la même trajectoire commerciale que la créature incarnée par Karloff, jusqu'à la reproduction en série d'objets dérivés qui en standardisent les traits; ce sont encore les traits schématisés de Lugosi que l'on retrouve, par exemple, chez le "Count Counting» dans la série télévisée pour enfant Sesame Street au début des années 1970. Les deux acteurs connaîtront une franche rivalité, exaltée, voire exagérée par les grands studios qui y voient le moyen de mousser leurs films ${ }^{14}$. Ils se sont par ailleurs échangé des rôles, ont partagé la vedette, ont été sacrés en même temps bogeymen. Mais alors que Lugosi s'est peu à peu identifié à son personnage de vampire, jusqu'à réclamer qu'on le prenne pour le comte Dracula, Karloff s'est admirablement résigné à jouer les seconds, loin derrière Frankenstein. C'est que Dracula était déjà, lui-même, acteur, à l'opposé, donc, de Frankenstein - entendu désormais au sens de la créature -, désarmant de franchise. Alors que Dracula a fini par produire une «attitude » sociale et mondaine dans laquelle s'est vue engloutie l'existence entière de Lugosi, Frankenstein s'en est tenu à la présentation brutale d'un faciès dont la seule présence explique l'horreur et le caractère exceptionnel. Si Karloff se confond avec Frankenstein,

12. Le nombre d'adaptations, de versions, de dérivés parodiques ou génériques est beaucoup trop élevé pour en permettre la nomenclature dans cet article. Peu de «héros » modernes connaissent une telle descendance. Le lecteur intéressé pourra s'en assurer en consultant internet, en particulier l'encyclopédie électronique «Wikipedia.org» et le site consacré au personnage «Frankenstein Castle». Ce dernier propose d'ailleurs, en ouverture, la formule suivante : «Frankenstein. A Monster with Many Faces». Il est frappant de constater combien la multitude des «faces » correspond, en réalité, à la multiplicité de répliques et de dérivés d’un visage unique. À de rares exceptions, le visage de Frankenstein rompt avec celui imposé par le film de Whale. Ce fut le cas en 1994, avec l'adaptation cinématographique proposée par Kenneth Branagh dans laquelle Robert De Niro tenait le rôle de la créature. L'entreprise consistait à reprendre le roman de Shelley afin de redonner à la créature son sens originel et à la trame narrative l'ampleur d'une tragédie. Malgré l'audace du film, le public eut du mal à relier le visage plutôt banal du monstre à celui, puissamment «imaginaire», qu'avait su créer Karloff.

13. Dracula, Tod Browning, Universal, 1931.

14. Gregory William Mank, Karloff and Lugosi. The Story of a Haunting Collaboration, Jefferson, North Carolina, McFarland \& Company, 1990. 
ce n'est donc qu'en tant que visage; et si ce visage a un effet de «masque », ce n'est pas au sens d'une cache ou d'un jeu, mais au sens plus archaïque d'une «statuaire» dont le caractère brut semble contredire les traces d'une fabrication. C'est la «stature », la «cadrure » qui marque les unes après les autres les diverses incarnations de Karloff et les nombreux dérivés du visage de Frankenstein. C’est cette cadrure qui renvoie à l'idée d'une pétrification d'un visage toujours déjà momifié. Si l'affaire Lugosi est celle d'un acteur qui finit par se prendre pour son personnage, l'affaire Karloff est celle d'un visage, uniquement: une image de visage qui n’arrive pas à sortir de son cadre et qui, pourtant, se prête à toutes les répétitions. Le «cadre », on l'aura compris, n'est pas seulement ici le «contexte» dans lequel est créé ce visage, mais le principe même de son exposition en tant qu'image en train de naître au sein même de sa propre mort. À mille lieues de l'élégance spectrale du vampire et de sa virtuosité toute «naturelle » qui en font, par excellence, le modèle du «beau parleur», le visage de Frankenstein s'expose, comme on le dirait d'un cadavre, muet et sans expressivité - une pure extériorité paradoxalement fermée sur elle-même. Pure extériorité, parce qu'on y saisit brutalement, à travers la décomposition des traits, les stigmates d'une expérience ratée, une écriture qui ne relate que sa propre entreprise à perte; fermée sur elle-même, parce que ce visage grège, marqué par l'occlusion chirurgicale, au lieu d'exprimer quelque chose, exhibe l'échec d'une naissance. En définitive, la «créature » n’appartient pas au registre habituel du mort-vivant, mais correspond plutôt à la vision que l'on pourrait avoir d'un avorton qui aurait survécu. La survivance est son mode d'être plutôt que la revenance.

Après des semaines de travail intense sur Boris Karloff (des centaines d'heures consacrées à la fabrication de ce visage), le maquilleur Jack Pierce du studio Universal en arrive au résultat que l'on connaît. Dans une livraison de la revue American Cinematographer datée de mai 1932, Pierce témoigne : il aura passé des mois à étudier les possibilités anatomiques du monstre. Chacun des effets des parties de visage, chaque relation de ces parties entre elles auraient été scrupuleusement étudiées: «Every line, every scar, every peculiarity of countour had to be just so for medical reasons; the eyes, for instance, were exact duplicates of the dead eyes of a 2,80o-year-old Egyptian corpse ${ }^{15}$. " Le visage de Frankenstein est donc un visage légendé: d'une part parce qu'il provient de la nuit des temps, conservé intact en son sarcophage; d'autre part parce qu'il porte sur lui, de manière ostentatoire, la genèse et l'explication de sa naissance. La naissance du monstre coïncide avec celle de sa légende, et sa fabrique se lit sur son visage - couches

15. Cité par Bryan Senn, Golden Horrors. An Illustrated Critical Filmography of Terror Cinema, 1931-1939, Jefferson, North Carolina, and London, McFarland \& Company Inc. Publishers, 1956, p. 22. 
successives, amoncelées, et pourtant toujours ostensibles, rendues au grand jour d'une mise en scène Grand-Guignol du maquillage. Sommet dans l'art de la tautologie, le grand œuvre de Pierce aura donc consisté à fabriquer la fabrication (sic!) d'un visage, à rendre l'artifice de la manière la plus authentique possible, mais aussi, à rendre ostensible une absence d'expressivité, voire une impossibilité à s'exprimer. Aux antipodes de la créature du roman de Shelley qui apprenait justement à parler, à lire et à écrire, le visage construit par Pierce ne peut que témoigner malgré lui d'un mutisme, d'une inadaptation au langage - témoignage rendu possible, néanmoins, à la faveur d'un effet de lecture. De manière encore plus frappante, le visage «expérimental» de la créature est décrété sans expérience, donc sans autre transmissibilité que son cadre de naissance, soit l'échec de sa mise au monde. L'image (en l'occurrence ce visage) signifie donc quelque chose, impose une lecture davantage qu'elle ne la requiert puisqu'elle agit sur le spectateur par l'effet d'un «surplus » de signes et de matière (coutures, stigmates, tracés, exposition délibérée d'un ouvrage raté $\left.{ }^{6}\right)$. Voilà par excellence un visage sans «essence» et sans vocation. La créature incarnée par Karloff ne peut donner lieu à aucun « portrait », car ce qu'il traîne partout comme on traîne ses oripeaux, c'est précisément une absence de vécu, voire le poids que peut constituer un interdit d'expérience. Si le personnage de Mary Shelley empruntait encore la voie d'une éducation sentimentale (à force de persévérance et d'attention au monde, il finit par se constituer une personnalité, un jugement, voire un certain talent dramatique), le Frankenstein joué par Karloff erre, pour ainsi dire, au milieu de ses propres velléités, sans évolution possible. Il n'arrive pas à s'émanciper de sa condition d'errance, comme le visage n'arrive pas à échapper à son cadre d'image. Est-ce également cette fameuse «carrure» qui dessine, non pas autour du visage, mais en lui-même un tel cadre - la merveille étant, en fait, que son créateur ait pourvu le monstre d'une immense boîte cranienne? La forme de cette boîte saisit le visage tout entier; elle l'« emboîte», le façonne complètement: les traits momifiés, le grain de peau crayeux, comme passé par la poussière et l'argile démiurgique, les paupières happées par les ombres, tout cela tombe sous le coup d'une forme vidée d'expressivité.

Mais qu'est-ce qu'un visage-cadre? Qu'est-ce qu'un visage enfermé dans son cadre d'image? C'est un visage sans extériorité ou, plutôt, dont l'extériorité s'est emparé de l'ensemble: tout est étranger à lui-même dans un tel visage, archicomposé, exposant sa fabrique, l'évidence de son aliénation. C'est une image qui ne peut donc circuler qu'en tant qu'image, dont la légende est celle de l'imagemonade, inapte: inapte à figurer, c'est-à-dire à passer du côté du langage, à engager des délibérations avec d'autres images (en effet, elle ne délibère pas avec

16. Voir Antoine de Baecque, «Le lieu à l'œuvre: fragments pour une histoire du corps au cinéma », Vertigo, no 15, 1996, p. 11-24. 
d'autres visages mais les contamine, les empèse, les bloque); inapte à s'émanciper du cliché, à s’approfondir. Les récits frankensteiniens sont toujours simples et répétitifs quand ils s'enferment dans ce visage karlovien. Si la postérité de Frankenstein passe par un visage, c'est donc parce que ce visage « retient», comme on retient la lumière, tout ce qui s'impose à lui. Rien ne peut l'altérer puisqu'il n'est déjà qu'altération.

Ce visage s'expose encore d'une autre manière: en projetant, dans l'industrie naissante du cinéma hollywoodien des années 1930, un vecteur singulier, celui des films d'horreur. Avec Frankenstein se déverse en effet un flot de visages jusque-là inconnu. Il suffit de prendre en considération les seules affiches de films de l'époque pour comprendre l'importance de ce phénomène. Les films qui s'affichent, et qui constituent ce nouveau genre horrifiant, ont pour carte de visite des visages (des gros plans de visages démesurés, fantastiques) : regard transperçant de Bela Lugosi en Dracula ou en zombie; faciès de monstres telluriques, de momies et de loups-garous ${ }^{17}$. Au cinéma, le visage, on le sait, est vendeur sous toutes les coutures, car les stars présentent elles aussi leur aura en s'affichant sur des panneaux géants - films d'amour, drames sentimentaux, aventures diverses. Mais ce qui fait la spécificité du genre de l'horreur, c'est qu'à chacun de ces visages en monstration correspond un mode d'être singulier et monadique. En feuilletant une telle collection, on est frappé par cette obsédante réitération de créatures à naître, de défigurations, de duplicités, d'ambivalences, d'inadéquations et de métamorphoses. Le cinéma hollywoodien s'empare d’une monstruosité trop humaine - de ce débordement d'humanité hors d'elle-même, de tout un peuple d'orphelins en marche: créatures errantes, vampires, momies, hommes-singes et zombies.

Les monades prolifèrent sous la forme de modes d'êtres inconciliables les uns avec les autres. Elles offrent alors au regard du spectateur des têtes, tantôt parlantes, tantôt bredouillantes, certes souvent inspirées par la littérature, mais détachées d'elle en tant qu'images. Car ce sont bien les images qui constituent le fond sur lequel s'articulent ces modes d'êtres. Et ces images, aussi monadiques soient-elles, se précipitent toutes vers des formes de sur-vivance: vie après la mort, mais surtout, vie en dehors de la vie, vie par-dessus les vivants, vie hors de la vie.

Norman M. Klein rappelait, dans un article consacré aux métamorphoses dans la culture américaine ${ }^{18}$, le «choc anamorphique» que constitue, dans la

17. Voir William K. Everson, «A Family Free of Monsters», Film Culture, vol. 1, $\mathrm{n}^{\circ} 1$, janvier 1955, p. 24-30.

18. Norman M. Klein, «Animation and Animorphs: A Brief Disappearing Act», dans Vivian Sobschack (dir.), Meta-morphing: Visual Transformation and the Culture of Quick-Change, Minneapolis, University of Minnesota Press, 2000, p. 30. 
culture urbaine américaine, l'immigration massive. Du point de vue de ceux qui sont nés à l'extérieur du pays et qui y vivent désormais, la culture américaine est en soi une culture de l'inadéquation. Par la force des choses, l'immigrant est sensible à toutes les difficultés d'apprentissage. Le rapport même à la langue produit son lot d'opacités et de régressions. Dans les années 1910-1940, on le sait, l'arrivée massive d'immigrants à Hollywood modifie considérablement l'art de faire des films et contribue à l'essor d'une véritable industrie dont on connaît aujourd'hui la force d'impact économique et idéologique. Rien d'étonnant à ce que le cinéma des années 1930 soit peuplé de visages rappelant la survivance, la transformation et la difficulté d'adaptation. L'extraordinaire force d'adaptation de plusieurs de ses artisans couvre l'anxiété avec laquelle la plupart des immigrants se voyaient projetés dans leur nouvelle vie. À l'excitation du «tout est possible» se lie l'appréhension de la perte et du sacrifice pour les générations à venir, celle également d'une difficile transmission de l'expérience.

Mais surtout, la crise économique de 1929 rend parfaitement actuel les motifs de l'inadéquation et de la survivance. Les différents vécus sont alors engloutis dans le désastre social; le lot quotidien des vies brisées, des familles défaites par les délocalisations et les relocalisations économiques, l’indigence et la nécessité de recommencer à nouveau, tout cela insuffle à l'imaginaire du monstre une fantaisie nouvelle. Les visages de l'horreur s'affichent avec autant d'assurance qu'ils exposent, eux aussi, la paradoxale condition de celui qui, forcé de renâ̂tre, rencontre dans sa nouvelle vie la démesure et le bannissement. À la figure destinale se substitue la condition de l'avorton. Ce sont aussi des visages sans nom ou dont le nom ne sera jamais «propre»; des visages à l'identité trouble, ravagée, voire invisible. Dans le contexte de telles transformations économiques et sociales, où se développe une culture du changement, les monstres peuvent aussi rassurer, en ce qu'ils sont les produits exacerbés de la transformation et les gardiens éternels du passé.

Boris Karloff et Bela Lugosi sont les produits de telles circonstances. Le nom de Karloff - à consonance russe, donc orientalo-européenne, donc lointaine, voire exotique pour un certain public - est un nom d'artiste, acquis dès ses débuts au théâtre par William Pratt, Anglais de naissance. Après des débuts au Canada, William débute une carrière à Hollywood en 1916. C'est toutefois Frankenstein qui propulse l'acteur à l'avant-plan et, avec lui, le nom de Karloff et, surtout, un visage. La légende s'empare alors de Karloff et lui assigne une origine tout aussi fantastique que celle de Frankenstein: selon la rumeur qui circule frénétiquement dans la presse de l'époque, Karloff serait le fils illégitime de l'amant égyptien de sa mère. La momie refait surface et, avec elle, le «destin» qui échappe au visage de Frankenstein. Ironiquement, le véritable nom de Jack Pierce, maquilleur-inventeur du visage qui nous occupe, est Janus 
Piccoulasvenou, émigrant d'origine grecque, venu en Californie depuis Chicago en 1910. Comme la plupart des artisans émigrés du cinéma hollywoodien, Pierce a occupé tous les emplois dans l'industrie: projectionniste, directeur de théâtre, caméraman, assistant-réalisateur et même acteur avant de joindre le studio Universal en 1926, comme maquilleur. Quant à Bela Lugosi, selon les différentes rumeurs intensément entretenues, il serait né tantôt en 1883, tantôt en 1889, plus vraisemblablement le 29 octobre 1882 en Hongrie. Il a joué des rôles mineurs dans plusieurs films allemands sous le nom de Arisztid Olt avant de poursuivre une carrière bien remplie aux États-Unis où il joua, entre autres, un pirate apache espagnol (sic), jusqu'à la consécration offerte par un Dracula sur Broadway à partir de 1927 et surtout, par la version cinématographique de Tod Browning en 1931. Pourquoi l'Europe de l'Est? Sans doute parce qu'elle représente alors la figure inversée de l'Europe civilisatrice: plus ancienne et plus nouvelle dans ses enfantements, espace imaginaire où s'enfoncent les ruines de l'Europe des Lumières. L'ambiguïté commune aux deux acteurs au sujet de leur naissance ne peut qu'alimenter mon propos. Certes, les prête-noms, les pseudonymes et autres noms d'artistes sont choses communes à l'époque. Mais la légende de ces deux acteurs, constamment confrontés l'un à l'autre, ajoute à l'indétermination identitaire, comme si le moment de la naissance devait appartenir, dans leur cas, au domaine de l'image pure, de la «fabrique d'images ». La trajectoire d'errance des acteurs participe du vecteur monadique du visage.

Comme le notait Bryan Senn, dans son ouvrage consacré à l'âge d'or de l'horreur à Hollywood ${ }^{19}$, le thème de la séparation entre «l'esprit» et «le bras " — thème séculaire s'il en est un —, est particulièrement important dans l'œuvre des deux acteurs. J'ajouterais: le «sur-vivant» est nécessairement fragmenté, brisé, inadéquat, parce qu'il est à la recherche infructueuse de ce qui pourrait lui procurer son sens de l'unité (un nom, une famille, un vécu). Or, la migration, l'implantation souvent forcée dans une autre culture ou dans une «autre vie» implique très souvent une inadéquation entre ce que commande l'ethos et l'affect. Alors que le sens de l'action et de la vie pratique engagent à la survie comme à la nouveauté de l'environnement, l'affect reconduit vers le territoire délaissé du passé. La légende véhiculée par les visages de l'horreur renverse et gonfle l'équation, car dans ces films, souvent considérés comme régressifs, c'est bien l'action qui est mal conduite, inadéquate, moralement répréhensible parce que tournée vers le passé, prise dans ses tares et dans d'irréparables injustices, alors que l'affect se rattache à la volonté de changement, au désir d'une impossible adaptation ou d'une improbable résolution des grands malheurs à travers elle (de la demande d'affection de Frankenstein à la mégalomanie de Dracula). Si

19. Voir Bryan Senn, Golden Horrors, p. 302. 
Lugosi meurt d'une adéquation trop parfaite entre lui-même et son rôle, Karloff cultive jusqu'à la fin l'inadéquation entre un visage d'horreur qu'il porte comme un talisman et la réputation d'un «ange». Dans un article datant de 1962 de la revue Bizarre consacrée à l'épouvante, Jean Boullet écrit à propos de Bela Lugosi et de Boris Karloff, «monstre malgré lui »: «Ils marchèrent, certes, la main dans la griffe, Karloff avec son auréole, sa trousse de secours et ses ailes de bon samaritain, Lugosi sentant le soufre à cent lieues, sans âme et sans reflet, traînant sa cape de magicien ornée d'une fantastique paire d'ailes de chauve-souris ${ }^{20} \ldots$ ». À cette allégorie fantastique s'oppose de manière significative la mise en abyme proposée par le film Targets, réalisé en 1968 par Peter Bogdanovich et mettant en vedette, pour la dernière fois, Boris Karloffer. Celui-ci y joue le rôle d'un acteur d'horreur vieillissant, confiné à la retraite et qui constate combien le quotidien peut dépasser en horreur les films dans lesquels il a joué. Le personnage principal du film, un jeune psychopathe au nom doublement suggestif de «Byron Orlok », tire à travers un écran de cinéma sur des spectateurs venus voir The Terror (Roger Corman), film d'horreur mettant en vedette Karloff lui-même en 1963 dans le rôle du Baron Von Leppe. Dans ces deux films, le visage de Karloff évoque encore celui de Frankenstein, au point où les affiches publicitaires en soulignent à l'excès les stigmates et la carrure. À travers cette scène de la tuerie, le testament filmique de Karloff fournit une spectaculaire mise en scène de l'adéquation - sublimation parfaite de l'inadéquation fondatrice mais combien fatale! En effet, l'horreur trouve son effectuation ultime à travers l'écran, littéralement, en réponse au vide existentiel d'un acteur à la retraite. Dans le film de Bogdanovich, le spectateur reçoit son dû «en plein visage » (c'est le cas de le dire), rendant pleinement effective l'idée selon laquelle le gros plan produit de la «visagéité ». Mais en l'occurrence, c'est bien à l'éclatement du visage, comme à celui de l'écran et de ce qui le sépare de son public, qu'invite le dernier film auquel Karloff prête son visage. Frankenstein-Karloff ne répond plus, comme chez Shelley, du mythe faustien ou prométhéen de l'irréparable expérience scientifique, mais de l'anxiété de l'inadéquation, une anxiété du quotidien, qui ne peut être résolue par la force réconciliatrice et synthétique d'une autorité narrative et de sa probité littéraire, mais qui persiste de manière monadique à travers l'affluence paradoxale d'un visage légendaire vidé de toute expérience.

20. Jean Boullet, «Boris Karloff, monstre malgré lui », Bizarre, $\mathrm{n}^{\mathrm{os}} 24-25,3^{\mathrm{e}}$ trimestre, 1962 , p. 75 .

21. Voir Isabel Pinedo, «Recreational Terror: Postmodern Elements of the Contemporary Horror Film », Journal of Film and Video, vol. 48, nos 1-2, printemps-été 1996, p. $17-31$. 